TIFR-TH-00/30

hep-th/0006043

\title{
NONCRITICAL STRINGS, RG FLOWS AND HOLOGRAPHY
}

\author{
Avinash Dhar* and Spenta R. Wadia ${ }^{\dagger}$ \\ Department of Theoretical Physics \\ Tata Institute of Fundamental Research, \\ Homi Bhabha Road, Mumbai 400 005, INDIA.
}

\begin{abstract}
We derive an RG flow equation that is satisfied by the regularized partition function for noncritical strings in background fields. The flow refers to change in the position of a "boundary" in the liouville direction. The boundary is required to regularize the ultraviolet divergences in the partition function coming from integration over world-sheets of arbitrarily small area. From the point of view of the target space effective gravitational action that the partition function evaluates on-shell, the boundary regularizes infrared divergences coming from the infinite volume of the liouville direction. The RG flow equation that we obtain looks very much like the Hamilton-Jacobi constraint equation that an on-shell gauge-fixed gravitational action must satisfy.
\end{abstract}

\footnotetext{
*adhar@tifr.res.in

† wadia@tifr.res.in
} 


\section{INTRODUCTION}

One of the central results that has emerged from the studies of the AdS/CFT correspondence [1, 2, 3] between 5-dimensional gravity in the AdS bulk and 4-dimensional Yang-Mills theory on its boundary is the identification of the renormalization scale of the latter with the radial coordinate of the AdS bulk and the radial evolution of the 5dimensional fields with RG flows of the couplings in the 4-dimensional Yang-Mills theory

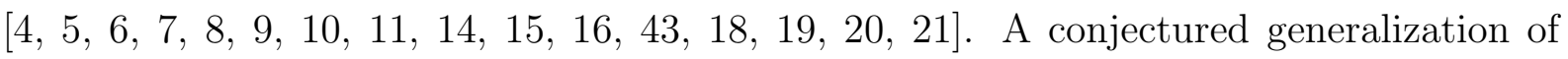
this correspondence to 4-dimensional boundary theories which include gravity [22, 23] has also recently been studied in the context of Randall-Sundram type brane-world scenarios [24, 25, 26, 27, 28] and the cosmological constant problem [29, 30, 31, 32.

The existence of a connection between RG flows in a D-dimensional theory, which includes gravity, and gravitational equations in $(\mathrm{D}+1)$-dimensions, was recognized and pointed out quite sometime back in the perturbative studies of noncritical string theory [33]. Perturbative noncritical string theory is formulated as D-dimensional matter coupled to 2-d quantum gravity [34. As is well-known, in this formulation of string theory the extra coordinate of the $(\mathrm{D}+1)$-dimensional space is related to the conformal degree of freedom of the world-sheet metric 35, 36, 37] and world-sheet gravitational dressing 38. of the various $\sigma$-model couplings gives rise to their dependence on this extra coordinate. A connection between the RG flows of the D-dimensional fields and gravitational equations in $(\mathrm{D}+1)$-dimensions arises 33 because the dependence of the $\sigma$-model couplings on the conformal mode of the world-sheet metric is determined by gravitational equations in $(\mathrm{D}+1)$-dimensions. In recent years this connection has been made precise in the context of AdS/CFT correspondence. The purpose of this note is to reexamine and further expand on the world-sheet approach of noncritical string theory to holographic RG in the light of these recent advances. The main advantage of this approach is that it provides a natural setting for the discussion of a generic holographic RG connection between D-dimensional boundary theories that contain gravity and $(\mathrm{D}+1)$-dimensional gravitational dynamics. 
The world-sheet approach also provides a systematic handle on stringy (i.e. $\alpha^{\prime}$ ) as well as string loop corrections.

The key organizing principle in the first quantized approach to noncritical string theory is the requirement of world-sheet reparametrization invariance. In the background gauge-fixing method, a prescription for integrating over the 2-d metric which ensures this requirement of reparametrization invariance automatically ensures Weyl invariance with respect to the 2-d fiducial metric. In this approach, therefore, Weyl invariance with respect to the 2-d fiducial metric emerges as the principal consistency requirement which is needed to ensure world-sheet reparametrization invariance. For example, it is this requirement that determines the gravitational dressing of the $\sigma$-model couplings.

In this note we will consider the $\sigma$-model partition function of noncritical strings propagating in background fields. This partition function is in general not well-defined because of divergent contributions to it arising from correlators of microscopic loop operators whose liouville wavefunctions are not normalizable. Although this is an ultraviolet (small area) divergence on the world-sheet, from the $(\mathrm{D}+1)$-dimensional target space point of view it is an infrared (large volume) divergence. It can be regularized by introducing a cut-off on the integration over the liouville zero mode. This is very much like the infrared regulator needed in the radial direction to evaluate the on-shell gravity action in AdS space. This way of regularizing the partition function introduces a "boundary" in the liouville direction. The regularized partition function depends on the location of the liouville boundary only implicitly through the values of the dressed couplings at the boundary. We will show here that a change in the location of the boundary gives rise to an RG flow equation for the partition function which looks exactly like the Hamilton-Jacobi constraint which an on-shell boundary gravitational action is expected to satisfy [39, 19].

The plan of this paper is as follows. In the next section we first summarize the main results [33] from the first quantized approach to noncritical string theory as Ddimensional matter coupled to 2-d gravity. We then discuss in detail the interpretation of the dependence of the $\sigma$-model couplings on the extra coordinate as RG dependence 
in the D-dimensional theory. In Sec. 3 we argue that the $\sigma$-model couplings should more correctly be interpreted as defining a boundary value problem in a $(\mathrm{D}+1)$-dimensional gravity theory. We explain how the boundary arises from the need to regularize worldsheet ultraviolet divergences in the calculation of the partition function. We then show that, consistent with its interpretation as an on-shell boundary action, the regularized partition function staisfies a flow equation which looks very much like a Hamilton-Jacobi constraint equation which an on-shell boundary gravitational action is expected to satisfy. We end in Sec. 4 with some concluding remarks.

\section{NONCRITICAL STRINGS AND HOLOGRAPHIC RG}

In this section we first briefly summarize some old and rather well-known results from noncritical string theory. We then discuss the RG scale dependence interpretation of the gravitational dressing of the couplings. For simplicity we restrict the discussion to bosonic string, but extension to superstring is straightforward.

The starting point of the first quantized approach to noncritical strings in background fields is the world-sheet reparametrization invariant action

$$
\begin{gathered}
S=\frac{1}{8 \pi \alpha^{\prime}} \int d^{2} \xi \sqrt{g}\left[\partial_{\alpha} X^{\mu} \partial_{\beta} X^{\nu}\left(g^{\alpha \beta} G_{\mu \nu}(X(\xi))+\epsilon^{\alpha \beta} B_{\mu \nu}(X(\xi))\right)\right. \\
\left.+\alpha^{\prime} R^{(2)} \Phi(X(\xi))+T(X(\xi))+\cdots\right]
\end{gathered}
$$

Here $x^{\mu}$ 's, which are the zero modes of $X^{\mu}(\xi)$ 's, parametrize a D-dimensional space with metric $G_{\mu \nu}(x)$ and other fields. The Polyakov path integral formally defines the partition function

$$
Z\left[G_{\mu \nu}, \Phi, B_{\mu \nu}, \cdots\right]=\int\left[\mathcal{D} g_{\alpha \beta}\right]\left[\mathcal{D} X^{\mu}\right] e^{-S}
$$

which is a functional of the D-dimensional couplings $G_{\mu \nu}, \Phi, B_{\mu \nu}$, etc. 


\section{Gravitational Dressing}

In the quantum theory the various $\sigma$-model couplings get dressed by 2-d gravity. A reparametrization invariant prescription for determining these gravitational dressings is the following. One first fixes the conformal gauge

$$
g_{\alpha \beta}=e^{\phi(\xi)} \hat{g}_{\alpha \beta} .
$$

Here $\phi(\xi)$ is the liouville mode and $\hat{g}_{\alpha \beta}$ is a fiducial metric that depends on the moduli of the Riemann surface over which the action in (2.1) is defined. One then makes a transformation in the functional integral from the liouville mode $\phi(\xi)$ to a field $\eta(\xi)$ with gaussian measure which, in the absence of the background fields, has the following action [40, 41]

$$
\frac{1}{8 \pi} \int d^{2} \xi \sqrt{\hat{g}}\left(\hat{g}^{\alpha \beta} \partial_{\alpha} \eta \partial_{\beta} \eta+Q \hat{R}^{(2)} \eta\right)
$$

where $Q=\sqrt{(25-D) / 3}$. When background fields are switched on, in the presence of 2-d gravity they get dressed, that is they become functions of $\eta$ [35, 33, 37]. Thus

$$
G_{\mu \nu}(x) \rightarrow G_{\mu \nu}(x, \eta), \quad \Phi(x) \rightarrow \Phi(x, \eta), \quad \cdots
$$

The $\eta$-dependence of the various fields is fixed by demanding that the above procedure preserve world-sheet reparametrization invariance. In particular, this means that the final results should be invariant under Weyl transformations of the fiducial metric $\hat{g}_{\alpha \beta}$. This leads to the familiar beta-function equations for the dressed fields

$$
\begin{aligned}
& 0=R_{M N}+2 \nabla_{M} \nabla_{N} \Phi-\frac{1}{4} H_{M P L} H_{N}{ }^{P L}+\cdots \\
& 0=\nabla^{P} H_{P M N}-2 \nabla^{P} \Phi H_{P M N}+\cdots \\
& 0=\frac{D-25}{3 \alpha^{\prime}}-R^{(D+1)}-4 \nabla^{P} \nabla_{P} \Phi+4 \nabla_{P} \Phi \nabla^{P} \Phi
\end{aligned}
$$




$$
+\frac{1}{12} H_{M N P} H^{M N P}+\cdots
$$

etc. Here the indices $M, N$ etc. run over $\mu, \eta$ and the dots represent $\alpha^{\prime}$ and string loop corrections. These equations have $(\mathrm{D}+1)$-dimensional general covariance.

\section{RG Flows}

Although we considered D-dimensional matter in the above discussion, these considerations actually apply to any matter coupled to 2-d gravity. A term in the action of the form

$$
\sum_{i} \int d^{2} \xi \sqrt{g} \lambda^{i} O_{i}\left(X(\xi), g_{\alpha \beta}(\xi)\right),
$$

where $O_{i}\left(X(\xi), g_{\alpha \beta}(\xi)\right)$ is a local operator constructed from the matter fields $\{X(\xi)\}$, gets dressed to

$$
\sum_{i} \int d^{2} \xi \sqrt{\hat{g}} \lambda^{i}(\eta(\xi)) O_{i}\left(X(\xi), \hat{g}_{\alpha \beta}(\xi)\right)
$$

and the $\eta$-dependence of the dressed coupling, $\lambda^{i}(\eta)$, is determined by an appropriate vanishing beta-function condition. In case the couplings $\lambda^{i}$ correspond to a CFT coupled to 2-d gravity, the $\lambda^{i}(\eta)$ are independent of $\eta$. This identifies CFT's as special points in the space spanned by the set of all possible couplings $\lambda^{i}$, the so-called theory space. The more general case in which the couplings get dressed can be interpreted as giving RG flows between these special points corresponding to CFT's.

It is important to emphasize here that the $\mathrm{RG}$ flow that we are talking about is not due to changes of cut-off in the 2-d QFT of $X(\xi)$ 's and $\eta(\xi)$. Although this cut-off is needed to do computations, the vanishing beta-function conditions ensure that the couplings do not depend on it. The RG flows that we are talking about are similar to finite size scaling. The size is here provided by the invariant area of the world-sheet, or its conjugate, the world-sheet cosmological constant [40, 41, 33]. In the conformal gauge, the flows thus correspond to the response of the couplings to changes of the physical scale brought about by shifts of the liouville mode, and hence of $\eta$. The vanishing beta-function equations 
describe how the dressed couplings change with precisely these shifts of $\eta$. In general, shifts of $\eta$ do not produce any simple scalings of the couplings, except near the points in theory space described by CFT's. The trajectories in theory space given by the dressed couplings describe RG flows between two such points. An example of such an RG flow between two $c<1$ minimal models has been discussed in detail in 33 where an explicit kink solution is given which describes RG flow due to a nearly marginal perturbation. I

For D-dimensional matter coupled to 2-d gravity, constant shifts of $\eta$ describe RG flows in a D-dimensional effective theory of gravity. To see this, consider the $(\mathrm{D}+1)$-dimensional gravity theory, obtained after dressing by 2-d gravity, in the gauge 2

$$
G_{\eta \eta}=1, \quad G_{\eta \mu}=0
$$

In this gauge, the $(\mathrm{D}+1)$-dimensional metric is given by

$$
d s_{D+1}^{2}=d \eta^{2}+d s_{D}^{2}
$$

where the metric in a constant $\eta \mathrm{D}$-dimensional slice is given by

$$
d s_{D}^{2}=G_{\mu \nu}(x, \eta) d x^{\mu} d x^{\nu}
$$

A shift in $\eta$ produces a change in the D-dimensional metric which is dictated by the $(\mathrm{D}+1)$-dimensional gravitational equations. In general, such a change generates a local change of scale in the D-dimensional world and hence corresponds to a local generalization of the usual RG flows. In the particular case that

$$
G_{\mu \nu}(x, \eta)=\Omega(\eta) G_{\mu \nu}(x)
$$

and $\Omega$ is a monotonic function of $\eta$, shifts in $\eta$ give global changes of scale in the D-

\footnotetext{
${ }^{1} \mathrm{RG}$ flows in two-dimensional theories coupled to gravity have also been considered in 42, 43].

${ }^{2}$ As we shall discuss in the next section, it is natural to guess that the partition function of noncritical strings in background fields evaluates a $(\mathrm{D}+1)$-dimensional gravitational action on-shell in this gauge.
} 
dimensional world and hence in this case we recover the standard RG flows in the Ddimensional theory.

\section{THE FLOW EQUATION}

In this section we will argue that the partition function for noncritical strings evaluates a $(\mathrm{D}+1)$-dimensional gravitational action on-shell, in the gauge (2.7), for solutions with a boundary. Consistent with this interpretation, we will derive a flow equation for the partition function, which looks just like the Hamilton-Jacobi constraint that the on-shell gauge-fixed (D+1)-dimensional action must satisfy for ensuring full $(\mathrm{D}+1)$-dimensional general covariance.

The partition function is formally given by the functional integral in (2.2) where $S$ is the action in (2.1). Now, it is well-known that the equations in (2.4)-(2.6) can be derived from a $(\mathrm{D}+1)$-dimensional gravitational action. Since these equations also determine the 2-d gravitational dressing of the $\sigma$-model couplings, it would seem consistent to identify the partition function in (2.2) with the $(\mathrm{D}+1)$-dimensional gravitational action evaluated on-shell. There is, however, a problem in this identification, which we will now discuss.

The set of equations (2.4)-(2.6) are partial differential equations of second or higher order, depending on the order to which terms are retained in $\alpha^{\prime}$ expansion. At the lowest order in $\alpha^{\prime}$, the equations are second order differential equations in $\eta$ for the dressed couplings. A general solution for these equations depends on two independent functions of $x$ for each of the background fields. For example, for the dilaton the general solution depends on $\Phi_{0}(x)$ and $\Phi_{0}^{\prime}(x)$, which may be taken to be respectively the value of the dressed field $\Phi(x, \eta)$ at some point $\eta=\eta_{0}$ and the value of its derivative $\partial_{\eta} \Phi(x, \eta)$ at $\eta_{0}$. Thus, at the lowest order in $\alpha^{\prime}$, the $(\mathrm{D}+1)$-dimensional on-shell action should depend on two independent functions of $x$ for each of the background fields. The partition function in (2.2), however, depends only on one function of $x$ for each of the background fields since its dependence on the background fields is inherited from the action in (2.1) which 
has this property ? $^{\text {. }}$

One might worry that the functional intergral in (2.2) defining the partition function for the noncritical string is only formal and that a more rigorous definition of this functional integral would show some subtility in counting the number of independent couplings. That in fact there is no proliferation of couplings in a more rigorous setting can be easily seen by reformulating (2.2) in the framework of dynamically triangulated random surfaces. Th Thus the partition function in (2.2) cannot be identified with a (D+1)-dimensional gravitational action evaluated on-shell for general solutions.

Actually, there are solutions to the differential equations (2.4)-(2.6) which depend on only one function of $x$ for each background field. These solutions involve (D+1)dimensional spaces with a D-dimensional boundary ?, and a regularity condition in the bulk generally picks up only one of the two possible (at the lowest order in $\alpha^{\prime}$ ) solutions which evolves the boundary data into the bulk. The $(\mathrm{D}+1)$-dimensional action evaluated on-shell for such solutions depends on only one function of $x$ for each of the background fields, which together constitute the boundary data.

A simple example of the above is provided by the coupling corresponding to the tachyon field in the noncritical string theory corresponding to the flat space linear dilaton solution of the equations (2.4)-(2.6). The gravitationally dressed tachyon coupling satisfies the following equation in this background [33, 35]:

$$
\left(\partial_{\eta}^{2}-Q \partial_{\eta}+\partial_{x}^{2}\right) T(x, \eta)-\frac{\partial V}{\partial T}=0
$$

where $V=-T^{2}+O\left(T^{3}\right)$ is the tachyon potential. Ignoring the cubic and higher order terms in the potential, and going to the momentum space conjugate to $x$, we get the two

\footnotetext{
${ }^{3}$ The situation becomes worse when higher order corrections in $\alpha^{\prime}$ are included in the differential equations in (2.4)-(2.6) since then the order in $\eta$ derivatives in these equations increases with a corresponding increase in the number of independent functions of $x$, for each background field, characterizing the general solution.

${ }^{4}$ See, for example, 44 .

${ }^{5}$ We will assume the D-dimensional boundary metric to have a Euclidean signature
} 
solutions

$$
\tilde{T}_{ \pm}(k, \eta)=e^{\frac{Q}{2} \eta} \psi_{ \pm, k}(\eta)
$$

where $\psi_{ \pm, k}(\eta)$ is the liouville wavefunction,

$$
\psi_{ \pm, k}(\eta)=e^{ \pm \eta \sqrt{\left(k^{2}-\frac{D-1}{12}\right)}} \psi_{ \pm, k}
$$

If we place a D-dimensional "boundary" in the liouville direction at $\eta_{0}$, we see that only the solution $\psi_{-}(\eta)$ is regular at $\eta \rightarrow+\infty$. The other solution, $\psi_{+}(\eta)$, is regular at $\eta \rightarrow-\infty$. The liouville wavefunctions for both these solutions are not normalizable. f

A more nontrivial example is provided by AdS gravity, which has been extensively studied recently in the context of the AdS/CFT correspondence. In this case the FeffermanGraham theorem [45] guarantees that there is a unique regular solution that evolves boundary data into the AdS bulk. The regular solution is, however, not normalizable [3, 2], just like the liouville wavefunctions for microscopic states in the above example of tachyon in flat space and linear dilaton background. For example, the regular solution to linearized equations for a scalar field in the AdS background behaves at large $r$ as $\lambda(x) r^{\Delta-D}$, where $\Delta$ is the dimension of the corresponding operator in the dual CFT description and $D$ is the dimension of the boundary. [7

We propose to identify the partition function in (2.2) with a "boundary" action evaluated on regular solutions obtained as described above. The boundary in $\eta$ is the Ddimensional space parametrized by the $x^{\mu}$ 's and the background fields appearing in the $\sigma$-model action $S$, (2.1), essentially account for the boundary values of the corresponding dressed fields. 0 Moreover, since no independent functions of $x$ corresponding to the com-

\footnotetext{
${ }^{6}$ For $D \geq 1$ this is true only if $k^{2} \geq \frac{D-1}{12}$, and then these wavefunctions correspond to microscopic states. Wavefunctions corresponding to operators with $k^{2}<\frac{D-1}{12}$ are normalizable and hence these correspond to macroscopic states. See, for example, 46. for a review of liouville theory.

${ }^{7}$ The other solution, which behaves at large $r$ as $r^{-\Delta}$, is normalizable.

${ }^{8}$ It is important to note here that the $\sigma$-model couplings are not equal to the values of the dressed couplings at the boundary. The former can, however, be traded for the latter once the dressings are known. The example of the dressing of the tachyon in noncritical string theory considered above provides
} 
ponents $G_{\eta \eta}$ and $G_{\eta \mu}$ of the (D+1)-dimensional metric appear in $S$, we propose that the partition function actually evaluates the boundary action in the gauge (2.7). As evidence for this we point out that, by construction, the partition function has D-dimensional general covariance, as is required by the second of the conditions in (2.7). A more non-trivial check for our proposal is provided by the first gauge-fixing condition which requires the partition function to satisfy a Hamilton-Jacobi type of constraint equation. Later in this section we will derive a flow equation for the partition function which has a remarkable resemblance to such an equation.

\section{The Liouville Boundary}

Let us first try to understand how a boundary arises in the liouville direction. As is well-known, in critical string theory, where the 2-d metric is non-dynamical, the background fields appearing in the $\sigma$-model action must satisfy the beta function equations for conformal invariance. In the noncritical formulation of string theory, however, the D-dimensional background fields appearing in (2.1) are completely arbitrary, since it is the integration over the liouville mode that now enforces conformal invariance. To make the discussion more general, let us rewrite (2.2) as follows:

$$
Z[\lambda]=\int\left[\mathcal{D} g_{\alpha \beta}\right] Z_{g}[\lambda]
$$

where

$$
Z_{g}[\lambda]=\int\left[\mathcal{D} X^{\mu}\right] \exp \left(\sum_{i} \int d^{2} \xi \sqrt{g} \lambda^{i}(X(\xi)) O_{i}\left(X(\xi), g_{\alpha \beta}(\xi)\right)\right)
$$

Here the set $\left\{O_{i}\right\}$ forms a complete basis of closed string operators and so the set of couplings $\left\{\lambda^{i}\right\}$ includes all the closed string modes. Now, from the point of view of the Ddimensional matter functional integral in (3.5), the 2-d metric is just an external fiducial

metric. As is well-known, in this case for generic couplings $\lambda^{i}, Z_{g}[\lambda]$ in (3.5) has a confora good illustration of this. The tachyon coupling that enters the $\sigma$-model is $\psi_{k}$ which appears on the right hand side of (3.3). This can clearly be traded for $\psi_{k}\left(\eta_{0}\right)$ using (3.3). 
mal anomaly. This anomaly has the effect that in the generic case all the couplings $\lambda^{i}$ get dressed by 2-d gravity. In perturbation theory, the liouville wavefunctions which give the dressings of the couplings are of two types, microscopic and macroscopic [46], depending on the operator they couple to. Since the wavefunctions which dress microscopic operators are not normalizable, integration over the liouville zero mode of correlation functions involving a sufficient number of these operators is generically divergent. This is a source of divergent contributions to the partition function, which thus needs a regulator to make it well-defined.

The dressing of the coupling corresponding to the dilaton operator plays a somewhat special role since this determines the effective string coupling. This can bring in other problems, so let us discuss the dressing of the dilaton coupling in some detail.

Let us first separate out the integral over the zero mode of $x$ in (3.5) and write

$$
Z_{g}[\lambda]=\int d^{D} x \mathcal{Z}_{g}[\lambda ; x]
$$

Now, let us consider the dilaton coupling. The dressing of this coupling is controlled by the corresponding beta function, which is essentially the matter central charge, and is determined by the condition that the total central charge of the matter plus 2-d gravity system should vanish. As a result when we fix the conformal gauge and change over from the liouville mode to the variable $\eta(\xi)$ in the functional integral of $\mathcal{Z}_{g}[\lambda ; x]$ over the 2-d metric, we pick up a linear term in the action for $\eta(\xi)$, similar to that in (2.3), but now with the coefficient $Q$ given by 47

$$
\begin{gathered}
Q=\left[\frac{25-D}{3}+\alpha^{\prime}\left(R^{(D)}+4 \nabla_{\mu} \nabla^{\mu} \Phi-4 \nabla_{\mu} \Phi \nabla^{\mu} \Phi-\frac{1}{12} H_{\mu \nu \lambda} H^{\mu \nu \lambda}\right)\right. \\
\left.+O\left(\alpha^{\prime 2}\right)\right]^{\frac{1}{2}}
\end{gathered}
$$

For flat space we recover the result in (2.3). In general there is a linear term in $\eta(\xi)$ even in critical dimensions. Also, since the other beta functions do not vanish for generic 
couplings, $Q$ is in general a function of $x$. The generic case is, therefore, difficult to deal with. However, since the couplings are arbitrary, we can choose them to be such that $Q$ is a real constant, I I independent of $x$, or at least sufficiently slowly varying with $x$ so that its dependence on $x$ can be ignored to the first approximation. Assuming this simplifying choice, we then have an effective string coupling that grows at one end of the $\eta$ direction. This is true even in critical dimensions. String perturbation theory, therefore, breaks down because of this strong-coupling singularity.

In some cases this strong-coupling singularity can be removed by generating a potential for $\eta(\xi)$ by switching on some additional backgrounds. For example, in the case of flat space in noncritical dimensions, a potential for $\eta(\xi)$ is generated by a 2-d cosmological constant term. We will assume here that we are dealing with such a case and an appropriate coupling has been switched on to remove the strong coupling singularity. However, even in cases where the strong-coupling singularity can be removed in this way, generically there is a divergence in the partition function (3.4) which comes from integration over the opposite end of the $\eta$ direction where the effective string coupling becomes arbitrarily weak. As we have already remarked, this is because of the contributions to the $\sigma$-model partition function of correlators involving microscopic loop operators whose liouville wavefunctions are not normalizable. Thus the partition function in (3.5) diverges for generic couplings, $\left\{\lambda^{i}\right\}$. Trom the world-sheet point of view this divergence is ultraviolet in nature because it comes from 2-d surfaces of small area. However, from the $(\mathrm{D}+1)$-dimensional point of view, this is an infrared divergence since it arises from the infinite volume in the (noncompact) liouville direction. One way of regulating this divergence is by introducing an appropriate cut-off on the integration over the zero mode of $\eta(\xi)$. This is how a "boundary" gets introduced in the $\eta$ direction, its location being at the value of the cut-off.

Once a boundary has been introduced in this way, we can trade-off the couplings

\footnotetext{
${ }^{9} Q$ real is needed to ensure a space-like interpretation for $\eta(\xi)$.

${ }^{10}$ This happens even when $Q$ in (3.7) vanishes.
} 
appearing in (3.4) for the boundary values of the dressed couplings. Now, as discussed in the previous section, a shift in the liouville mode generates a local scale transformation on the boundary. Therefore, a shift of the cut-off generates an RG flow in the regularized partition function through the boundary values of the dressed couplings, leading to a flow equation which we will now derive.

\section{The Equation}

After fixing the conformal gauge and transforming from the liouville mode to the variable $\eta(\xi)$, the partition function in (3.4) may be written as

$$
Z\left[\lambda ; \eta_{0}\right]=\int d^{D} x \mathcal{Z}\left[\lambda ; \eta_{0} ; x\right]
$$

where

$$
\mathcal{Z}\left[\lambda ; \eta_{0} ; x\right]=\int_{\eta_{0}}^{\infty} d \eta \mathcal{L}[\lambda(\eta) ; x]
$$

and $\eta$ is the zero mode of $\eta(\xi)$ with $\eta_{0}$ the cut-off or the boundary value. On the right hand side of (3.9) we have made it explicit that the $\eta$-dependence comes entirely from the dressings of the couplings.

The flow equation can now be derived by making an $x$-dependent change in $\eta_{0}$, namely $\eta_{0} \rightarrow \eta_{0}+\epsilon(x)$. I Denoting the value of the dressed coupling at the boundary by $\lambda_{0}^{i}(x)$, the flow equation is

$$
\mathcal{L}\left[\lambda\left(\eta_{0}\right) ; x\right]=\partial_{\eta_{0}} \lambda_{0}^{i}(x) \frac{\delta Z}{\delta \lambda_{0}^{i}(x)}
$$

This equation follows from the fact that a change in $\mathcal{Z}\left[\lambda ; \eta_{0} ; x\right]$ produced by a shift in $\eta_{0}$ can be computed in two different ways. One is directly from the way $\eta_{0}$ appears as

\footnotetext{
${ }^{11}$ This $x$-dependent change in $\eta_{0}$ is made possible by the fact that we could have chosen an $x$-dependent cut-off on $\eta$. This possibility of a local cut-off on $\eta$ is compatible with the requirement of world-sheet reparametrization invariance.
} 
an integration limit in (3.9). This gives the left hand side of the equation. The other is by recognizing that $\mathcal{Z}\left[\lambda ; \eta_{0} ; x\right]$ depends on $\eta_{0}$ only through the boundary values of the dressed couplings since the $\eta$ dependence in $\mathcal{L}[\lambda(\eta) ; x]$ comes entirely from the liouville dressings of the couplings and the subsequent transformation to the gaussian variable $\eta$. This gives the right hand side of the equation.

At the lowest order in $\alpha^{\prime}$, we expect $\mathcal{L}[\lambda(\eta) ; x]$ to be only quadratic in $\eta$-derivatives of the dressed couplings. Let us write this out explicitly as $\square$

$$
\mathcal{L}[\lambda(\eta) ; x]=\frac{1}{2} \mathcal{G}_{i j} \partial_{\eta} \lambda^{i}(x, \eta) \partial_{\eta} \lambda^{j}(x, \eta)+V[\lambda(\eta) ; x]
$$

where $\lambda^{i}(x, \eta)$ is the dressed coupling, $\mathcal{G}^{i j}$ is

the metric on the space of the couplings and $V$ is assumed to have a local expansion in $x$-derivatives of $\lambda^{i}(x, \eta)$. We have made the reasonable assumption that $\mathcal{L}$ has a low energy expansion in derivatives of $\eta$ and $x$. Once (3.11) is given, one can show that the variation of the partition function with respect to the boundary values of the dressed couplings is related to the "velocities" in the standard way,

$$
\frac{\delta Z}{\delta \lambda_{0}^{i}(x)}=\mathcal{G}_{i j} \partial_{\eta_{0}} \lambda_{0}^{j}(x) .
$$

The flow equation (3.10) may then be rewritten as

$$
\frac{1}{2} \mathcal{G}^{i j} \frac{\delta Z}{\delta \lambda_{0}^{i}(x)} \frac{\delta Z}{\delta \lambda_{0}^{j}(x)}=V\left[\lambda_{0} ; x\right]
$$

which is the advertized Hamilton-Jacobi type of constraint equation that the regularized partition function must satisfy.

\footnotetext{
${ }^{12}$ We may assume the standard normalization for the "kinetic" term without any loss of generality. A possible linear term in $\lambda^{i}(x, \eta)$ can be removed by a field redefinition of the original sigma-model couplings $\lambda^{i}$. We will assume that this has been done and that the couplings $\lambda^{i}$ have been chosen accordingly. Also, note that the right hand side of (3.11) is evaluated on-shell in the sense that the $\eta$-dressing of the various couplings is determined by the requirement of reparametrization invariance. We also mention that the form of $\mathcal{L}$ assumed in (3.11) can be derived close to a CFT point in theory space.
} 


\section{CONCLUDING REMARKS}

In this note we have presented noncritical string theory as a boundary value problem, based on the observation that the liouville or conformal mode gives rise to an additional dimension. As we have argued, the boundary arises from the cut-off needed to regulate world-sheet ultraviolet divergences. We have shown that, under some reasonable assumptions, the partition function of the noncritical string $\sigma$-model action satisfies a Hamilton-Jacobi type of constraint equation as a functional of the boundary values of the $\sigma$-model couplings. The dependence of the couplings on the additional dimension is determined by the first order local RG flow equations (3.12). These equations were obtained for the bosonic string, but extension to the superstring is straightforward when RR backgrounds are absent. Since RR backgrounds couple to bilinears of space-time fermions in the $\sigma$-model, the analysis becomes complicated when these backgrounds are switched

on [48]. For this reason it is difficult to demonstrate explicitly that a Hamilton-Jacobi type of constraint equation continues to be satisfied in the presence of RR backgrounds, although we expect this to be the case. Finally we mention that the structure of the solution space of the RG flow equations (3.12) is presently not known 32. In order to address this issue it would be worthwhile to discuss the global topology of the RG flows along the lines presented in 490 where the global topology of a class of $c<1$ models was exactly calculated using methods of Morse theory.

\section{Acknowledgements}

One of us (SRW) would like to thank Theory Division, CERN, for hospitality during a visit when part of this work was done. 


\section{References}

[1] J. Maldacena, Adv. Theor. Math. Phys 2 (1998) 231, hep-th/9711200.

[2] S.S. Gubser, I.R. Klebanov and A.M. Polyakov, Phys. Lett. B428 (1998) 105, hepth/9802109].

[3] E. Witten, Adv. Theor. Math. Phys 2 (1998) 253, hep-th/9802150].

[4] E.T. Akhmedov, Phys. Lett. B442 (1998) 152, hep-th/9806217.

[5] E. Alvarez and C. Gomez, Nucl. Phys. B541 (1999) 441, [hep-th/9807226].

[6] V. Balasubramanian, P. Kraus and A. Lawrence, Phys. Rev. D59 (1999) 046003, hep-th/9805171.

[7] V. Balasubramanian, P. Kraus, A. Lawrence and S.P. Trivedi, Phys. Rev. D59 (1999) 104021, hep-th/9808017.

[8] L. Girardello, M. Petrini, M. Porrati and A. Zaffaroni, JHEP 9812 (1998) 022, hep-th/9810126.

[9] J. Distler and F. Zamora, hep-th/9810206.

[10] A. Khavaev, K. Pilch and N.P. Warner, hep-th/9812035.

[11] A. Karch, D. Lust and A. Miemiec, Phys. Lett. B454 (1999) 152, hep-th/9901041.

[12] V. Balasubramanian and P. Kraus, Phys. Rev. Lett. 83 (1999) 3605, hepth/9903190].

[13] M. Porrati and A. Starinets, Phys. Lett. B454 (1999) 77, [hep-th/9903241].

[14] I. Klebanov and E. Witten, Nucl. Phys. B556 (1999)89, hep-th/9905104].

[15] D.Z. Freedman, S.S. Gubser, K. Pilch and N.P. Warner, hep-th/9906194.

[16] L. Girardello, M. Petrini, M. Porrati and A. Zaffaroni, hep-th/9909047. 
[17] K. Skenderis and P.K. Townsend, hep-th/9909070.

[18] O. DeWolfe, D.Z. Freedman, S.S. Gubser and A. Karch, hep-th/9909134.

[19] J. De Boer, E. Verlinde and H. Verlinde, hep-th/9912012.

[20] J. Polchinski and M. Strassler, hep-th/0003136.

[21] C. Schmidhuber, hep-th/9912155.

[22] L. Randall and R. Sundram, hep-th/9905221 and hep-th/9906064.

[23] H. Verlinde, hep-th/9906182.

[24] S.S. Gubser, hep-th/9912001.

[25] V. Sahakian, hep-th/0002126.

[26] R. Gregory, V.A. Rubakov and S.M. Sibiryakov, hep-th/0002072.

[27] C. Csaki, J. Erlich and T.J. Hollowood, hep-th/0002161.

[28] G. Dvali, G. Gababadze and M. Porrati, hep-th/0002190.

[29] N. Arkani-Hamed, S. Dimopoulos, N. Kaloper, and R. Sundram, hep-th/0001197.

[30] S. Kachru, M. Schulz and E. Silverstein, hep-th/0001206.

[31] E. Verlinde and H. Verlinde, hep-th/9912018.

[32] S.S. Gubser, hep-th/0002160.

[33] S.R. Das, A. Dhar and S.R. Wadia, Mod. Phys. Lett. A5 (1990) 799.

[34] A.M. Polyakov, Phys. Lett. B103 (1981) 207, 211.

[35] S.R. Das, S. Naik and S.R. Wadia, Mod. Phys. Lett. A4 (1989) 1033.

[36] T. Banks and J. Lykken, Nucl. Phys. B331 (1990) 173. 
[37] A. Dhar, T. Jayaraman, K.S. Narain and S.R. Wadia, Mod. Phys. Lett. A5 (1990) 863.

[38] V. Knizhnik, A. Polyakov and A. Zamolodchikov, Mod. Phys. Lett. A3 (1988) 819.

[39] A.M. Polyakov, hep-th/9304146.

[40] F. David, Mod. Phys. Lett. A3 (1988) 1651.

[41] J. Distler and H. Kawai, Nucl. Phys. B321 (1989) 509.

[42] I.R. Klebanov, I.I. Kogan and A.M. Polyakov, Phys. Rev. Lett. 71 (1993) 3243, [9309106].

[43] C. Schmidhuber and A.A. Tsyetlin, Nucl. Phys. B426 (1994) 187, hep-th/9312155].

[44] V. Kazakov and A.A. Migdal, Nucl. Phys. B311 (1988) 171.

[45] C. Fefferman and C.R. Graham, in Elie Cartan et les Mathematiques d'Aujourdhui (Asterisque, 1985) 95.

[46] N. Seiberg, in Random Surfaces and Quantum Gravity, Eds. O. Alvarez, E. Marinari and P. Windey, NATO ASI series, Vol.262 (Plenum, 1991) 363.

[47] C.G. Callan, D. Friedan, E.J. Martinec and M.J. Perry, Nucl. Phys. B262 (1985) 593.

[48] N. Berkovits, C. Vafa and E. Witten, JHEP 9903 (1999) 018, hep-th/9902098.

[49] S.R. Das, G. Mandal and S.R. Wadia, Mod. Phys. Lett A4 (1989) 745. 